\title{
Umklapp induced surface band structure of $\mathrm{Ag} / \mathrm{Ge}(111) 6 \times 6$
}

Hafiz Muhammad Sohail and Roger Uhrberg

The self-archived postprint version of this journal article is available at Linköping University Institutional Repository (DiVA):

http://urn.kb.se/resolve?urn=urn:nbn:se:liu:diva-116940

N.B.: When citing this work, cite the original publication.

Muhammad Sohail, H., Uhrberg, R., (2015), Umklapp induced surface band structure of Ag/Ge(111)6 x 6, Surface Science, 635, 55-60. https://doi.org/10.1016/j.susc.2014.12.008

Original publication available at:

https://doi.org/10.1016/j.susc.2014.12.008

Copyright: Elsevier

http://www.elsevier.com/

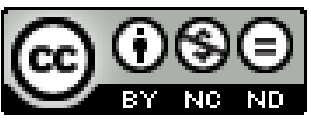




\author{
Umklapp induced surface band structure of $\mathrm{Ag} / \mathrm{Ge}(111) 6 \times 6$ \\ Hafiz M. Sohail* and R. I. G. Uhrberg \\ Department of Physics, Chemistry and Biology, Linköping University, S-581 83 Linköping, Sweden
}

\begin{abstract}
This study focuses on the electronic structure of a $6 \times 6$ surface which is formed by 0.2 monolayer of Ag on top of the Ag/Ge(111) $\sqrt{3} \times \sqrt{3}$ surface. The $6 \times 6$ periodicity was verified by low energy electron diffraction. Angle resolved photoelectron spectroscopy was employed to study the electronic structure along the $\bar{\Gamma}-\bar{M}-\bar{\Gamma}$ and $\bar{\Gamma}-\bar{K}-\bar{M}$ high symmetry lines of the $6 \times 6$ surface Brillouin zone. There are six surface bands in total. Out of these, three were found to be related to the $6 \times 6$ phase. The surface band structure of the $6 \times 6$ phase is significantly more complex than that of the $\sqrt{3} \times \sqrt{3}$ surface. This is particularly the case for the uppermost surface band structure which is a combination of a surface band originating from the underlying $\sqrt{3} \times \sqrt{3}$ surface and umklapp scattered branches of this band. Branches centered at neighboring $6 \times 6$ SBZs cross each other at an energy slightly below the Fermi level. An energy gap opens up at this point which contains the Fermi level. The complex pattern of constant energy contours has been used to identify the origins of various branches of the surface state dispersions.
\end{abstract}

Keywords: Ge(111), silver (Ag), electronic band structure, umklapp bands, LEED, and ARPES.

* Corresponding author:

Hafiz M. Sohail

Email: sohail@ifm.liu.se 


\section{Introduction}

Metal adsorbates on semiconductor surfaces are important since various one dimensional (1D) and two dimensional (2D) structures can be formed. Some of these act as simple model systems for the study of various physical phenomena [1-6]. Extensive studies have been performed on adsorbate induced structures on, for instance, $\mathrm{Si}(111)$ and $\mathrm{Ge}(111)$. Some of these structures form on top of the (111) crystals terminated by an atomic double layer, while others form on a modified crystal where the outermost atomic layer is missing. Silver (Ag) and gold (Au) are examples of metals that induce various 2D structures on $\mathrm{Si}(111)$ and $\mathrm{Ge}(111)$ where the outermost layer is missing [7-14], as in the case of the $\sqrt{3} \times \sqrt{3}$ structure formed by one monolayer (1 ML) of either Au or Ag [7,8]. These $\sqrt{3} \times \sqrt{3}$ surfaces have been subjected to intensive studies because of their interesting electronic and atomic structures [1, 15-27]. Further, an addition of noble ( $\mathrm{Ag}, \mathrm{Au}$ and $\mathrm{Cu}$ ) or alkali (Cs, $\mathrm{K}$ and $\mathrm{Na}$ ) metal atoms results in new periodicities that are formed as superstructures on the $\sqrt{3} \times \sqrt{3}$ surface. They develop after the addition of these extra atoms without annealing, as the atoms find specific sites on the $\sqrt{3} \times \sqrt{3}$ surface lattice. The $\sqrt{21} \times \sqrt{21}$ and $6 \times 6$ periodicities formed by small amounts of monovalent atoms (Ag, $\mathrm{Au}, \mathrm{Cu}, \mathrm{Cs}$, $\mathrm{K}$ or $\mathrm{Na}$ ) added to the $\mathrm{Ag} / \mathrm{Si}(111) \sqrt{3} \times \sqrt{3}$ surface are two such examples [9,28-32]. Similarly, a small amount of $\mathrm{Ag}$ or $\mathrm{Au}$ on $\mathrm{Ag} / \mathrm{Ge}(111) \sqrt{3} \times \sqrt{3}$ results in $\sqrt{39} \times \sqrt{39}$ and $6 \times 6$ superstructures [10,33-35].

In this paper, we present a detailed electronic structure study of the $6 \times 6$ phase prepared by depositing 0.2 ML of Ag onto a well-ordered $\mathrm{Ag} / \mathrm{Ge}(111) \sqrt{3} \times \sqrt{3}$ surface at room temperature (RT). The $6 \times 6$ surface shows a very rich surface band structure as revealed by angle resolved photoelectron spectroscopy (ARPES) along the $\bar{\Gamma}-\bar{M}-\bar{\Gamma}$ and $\bar{\Gamma}-\bar{K}-\bar{M}$ high symmetry lines of the 6×6 surface Brillouin zone (SBZ). The uppermost surface bands of the rather complex band structure can be identified as originating from umklapp scattering of a parabolic-like metallic surface band of the Ag/Ge(111) $\sqrt{3} \times \sqrt{3}$ surface. The hybridization of the parabolic bands centered at different $\bar{\Gamma}$-points of the $6 \times 6$ SBZs leads to gaps in the surface band structure.

\section{Experimental details}

ARPES was employed to study the electronic structure of the $6 \times 6$ surface. Experiments were performed at beamline I4, at the MAX-III storage ring of the MAX-lab synchrotron 
radiation facility in Lund, Sweden. Photoemission data were obtained at a photon energy of 30 $\mathrm{eV}$ with energy and angular resolutions of $\approx 50 \mathrm{meV}$ and $\pm 0.3^{\circ}$, respectively. A hemispherical electron analyzer with a 2D detector (SPECS Phoibos 100) was used to collect the electronic structure data consisting of emission intensity versus emission angle maps. Low energy electron diffraction (LEED) was used to check the surface quality and to align the sample azimuthally. The vacuum system had a base pressure of less than $1 \times 10^{-10}$ Torr, while Ag depositions were made at pressures of $<4 \times 10^{-10}$ Torr. A sample was cut from an n-type (Sb-doped) Ge(111) wafer with a resistivity in the range 7-10 $\Omega \mathrm{cm}$ at RT. Ultrasonic cleaning of the $\mathrm{Ge}(111)$ sample in acetone and isopropanol was done ex-situ. In-situ cleaning was performed by repeated $\mathrm{Ar}^{+}$ion sputtering $(1 \mathrm{keV})$ and annealing cycles $\left(\approx 730{ }^{\circ} \mathrm{C}\right)$, which resulted in a well-ordered $\mathrm{Ge}(111) \mathrm{c}(2 \times 8)$ surface as verified by LEED. The Ag evaporation rate was established using a quartz crystal thickness monitor. First, 1.1 ML was deposited at a rate of $0.5 \mathrm{ML} / \mathrm{min}$, where one ML is defined by the density of atoms on an unreconstructed $\mathrm{Ge}(111)$ surface (i.e., $7.2 \times 10^{14}$ atoms $/ \mathrm{cm}^{2}$ ). Annealing of the surface up to $330{ }^{\circ} \mathrm{C}$ for a few minutes, resulted in a well-ordered $\mathrm{Ag} / \mathrm{Ge}(111) \sqrt{3} \times \sqrt{3}$ superstructure. An initial amount of $1.1 \mathrm{ML}$ ensures that the surface has a full 1 ML coverage of Ag and that there are no $c(2 \times 8)$ or $4 \times 4$ domains, as was verified by LEED. The $\mathrm{Ag} / \mathrm{Ge}(111) 6 \times 6$ phase was prepared at RT by depositing 0.2 ML of $\mathrm{Ag}$ onto the $\mathrm{Ag} / \mathrm{Ge}(111)$ $\sqrt{3} \times \sqrt{3}$ surface.

\section{Results and discussion}

In Figs. 1(a) and 1(b), we present LEED results for the $\sqrt{3} \times \sqrt{3}$ and $6 \times 6$ surfaces, respectively. Fig. 1(a) shows only sharp diffraction spots which verifies that the surface has a well-ordered $\sqrt{3} \times \sqrt{3}$ periodicity. The absence of other diffraction spots implies that the surface is homogeneously covered by the $\sqrt{3} \times \sqrt{3}$ phase without patches of $4 \times 4$ or the initial $c(2 \times 8)$ reconstruction that are present at a coverage of less than $1 \mathrm{ML}[7,12,13]$. Furthermore, since there is no ring-like diffraction around the sharp spots, which would be indicative of a small surplus of Ag, we conclude that the amount of Ag remaining after annealing must be very close to $1 \mathrm{ML}$. However, after adding a small amount of Ag (0.2 ML), there are extra spots which correspond to a $6 \times 6$ periodicity, see Fig. 1(b). 

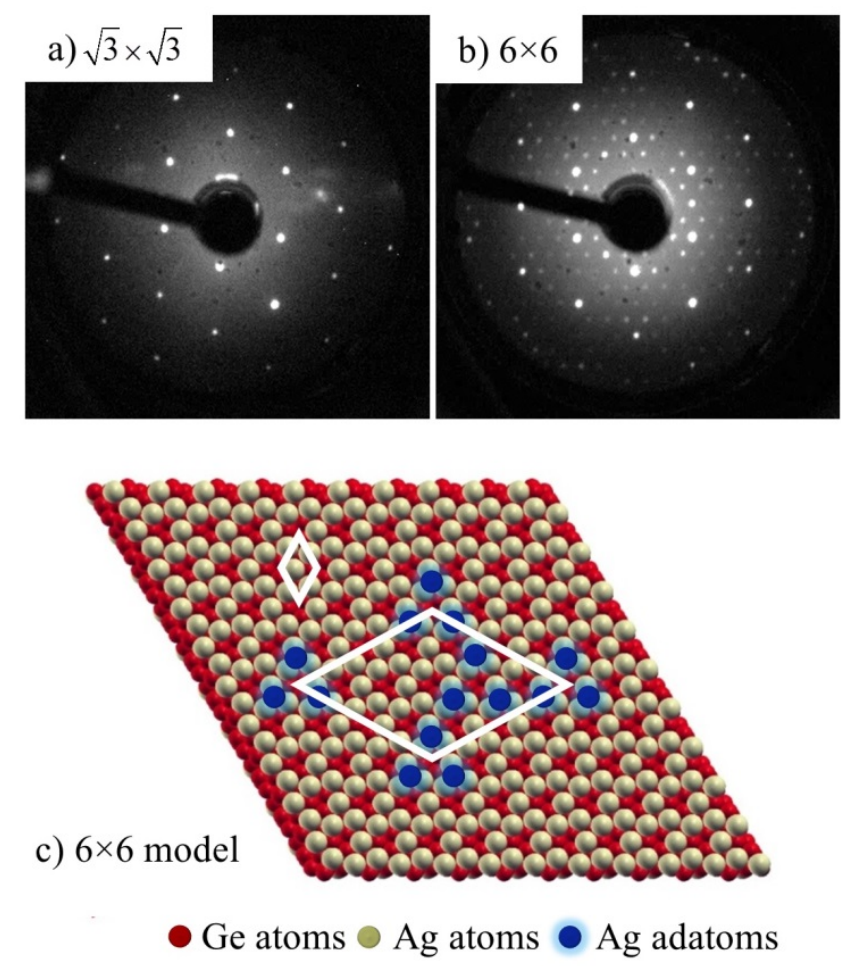

Fig. 1 (a) and (b) LEED patterns obtained at an electron energy of $60 \mathrm{eV}$ from the $\mathrm{Ag} / \mathrm{Ge}(111) \sqrt{3} \times \sqrt{3}$ and $\mathrm{Ag} / \mathrm{Ge}(111) 6 \times 6$ surfaces, respectively. (c) Atomic model of the 6×6 surface derived from Refs. 13, 23, and 35. The $6 \times 6$ periodicity is formed by $1 / 6 \mathrm{ML}$ of $\mathrm{Ag}$ positioned on top of the $\sqrt{3} \times \sqrt{3}$ surface. The small and the large parallelograms represent the unit cells of the $\sqrt{3} \times \sqrt{3}$ and the $6 \times 6$ periodicities, respectively.

The $\sqrt{3} \times \sqrt{3}$ spots, which are part of the $6 \times 6$ pattern, are strong which indicates that the initial surface is not destroyed and that the additional Ag atoms are positioned at specific $\sqrt{3} \times \sqrt{3}$ lattice sites to form the $6 \times 6$ periodicity. An atomic model of the $6 \times 6$ surface is shown in Fig. 1(c), which was derived from earlier STM images and suggested models in Refs. 13, 23, and 35. The STM images presented in Refs. 23 and 35 revealed a $6 \times 6$ structure in which six bright protrusions per $6 \times 6$ unit cell could be clearly seen. These protrusions were interpreted as extra Ag atoms. Three of the six Ag atoms form a triangle inside the cell that is slightly larger in size compared to the triangles located at the corners of the $6 \times 6$ unit cell formed by the remaining three Ag atoms, see Refs. 23 and 35. Furthermore, six extra Ag atoms per 6×6 unit cell correspond to $1 / 6 \mathrm{ML}$ which is consistent with the $0.2 \mathrm{ML}$ of $\mathrm{Ag}$ added to the $\sqrt{3} \times \sqrt{3}$ surface.

The electronic structure of the Ag/Ge(111)6×6 surface was studied by ARPES, using linearly polarized synchrotron light at an energy of $30 \mathrm{eV}$. The measurements were performed at 
RT. In Fig. 2, the experimental band structures of the $\sqrt{3} \times \sqrt{3}$ and $6 \times 6$ surfaces are presented. Figure 2(a) shows $1 \times 1$ (black hexagon), $\sqrt{3} \times \sqrt{3}$ (blue hexagons) and $6 \times 6$ (red hexagons) SBZs and highlights the high symmetry directions. The region within the black rectangle in Fig. 2(a) was mapped to obtain the 2D contour plots shown in Fig. 3. In Figs. 2(b) - 2(e), we present the second derivative, along the energy axis, of the original photoemission data. Dark features represent the positions of photoemission structures as function of energy and $k_{\square}$. Using the second derivative to display the data makes also weak but well-defined structures visible. The dashed curves in Figs. 2(b) - 2(e) represent the edge of the projected bulk bands, while the vertical dashed lines mark high symmetry points. Figures 2(b) and 2(c) show the experimental band structure of the $\sqrt{3} \times \sqrt{3}$ surface, along the $\bar{\Gamma}-\bar{K}-\bar{M}$ and the $\bar{\Gamma}-\bar{M}-\bar{\Gamma}$ high symmetry lines of the $\sqrt{3} \times \sqrt{3}$ SBZ, respectively, see the blue hexagons and the green and red lines in Fig. 2(a). There are four surface bands indicated by $\mathrm{S}_{1}-\mathrm{S}_{4}$ in Figs. 2(b) and 2(c), which are all within the projected bulk band gap. $S_{1}$, which is a free electron like band with a parabolic shape, and the $S_{2}$ - $\mathrm{S}_{4}$ surface bands have been studied in detail in Ref. 36. Figures 2(d) and 2(e) present the band structure of the $6 \times 6$ surface along $\bar{\Gamma}-\bar{K}-\bar{M}$ and $\bar{\Gamma}-\bar{M}-\bar{\Gamma}$ of the $6 \times 6$ SBZ, respectively, see the red hexagons and the green and red lines in Fig. 2(a). There are six surface bands in total of which some follow the $6 \times 6$ periodicity, and all are in the gap region within $1.8 \mathrm{eV}$ below the Fermi level $\left(E_{F}\right)$. The $S_{2}-S_{4}$ bands of the $\sqrt{3} \times \sqrt{3}$ surface are present also in the data from the $6 \times 6$ surface with just small changes in their appearance. The dispersion of the $\mathrm{S}_{1}$ band, on the other hand, undergoes dramatic changes. $\mathrm{S}_{1}$ on the $\sqrt{3} \times \sqrt{3}$ surface is an essentially empty parabolic band that has the energy minimum just slightly below $\mathrm{E}_{\mathrm{F}}$. The precise position of the minimum is very sensitive to electron doping caused by electron donation from a small amount of additional Ag atoms on the surface. One should also note that the minimum of the $\mathrm{S}_{1}$ band is located at $\bar{\Gamma}$ points of the $\sqrt{3} \times \sqrt{3}$ SBZ. Experimentally, it is difficult to identify $S_{1}$ in normal emission, but it appears clearly at $\bar{\Gamma}$ - points of the $\sqrt{3} \times \sqrt{3}$ SBZ coinciding with $\bar{K}$-points of the $1 \times 1 \mathrm{SBZ}$. In the $\sqrt{3} \times \sqrt{3}$ data presented in Fig. 2(b) the $\mathrm{S}_{1}$ minimum is at $\approx-0.65 \mathrm{eV}$ at the $\bar{\Gamma}_{1}$ - point of the $\sqrt{3} \times \sqrt{3}$ $\operatorname{SBZ}\left(k_{\square}=1.05 \AA^{-1}\right)$.

When more $\mathrm{Ag}$ is added to the $\sqrt{3} \times \sqrt{3}$ surface, to form the $6 \times 6$ surface, the minimum moves to higher binding energy. Comparing Figs. 2(b) and 2(d), one finds that the minimum of 
$\mathrm{S}_{1}$ at $k_{1}=1.05 \AA^{-1}$ has moved downward to $\approx-0.85 \mathrm{eV}$. However, a more striking difference is the presence of several new bands in the energy region of $S_{1}$. There are no bands in the $S_{1}$ region in the $\sqrt{3} \times \sqrt{3}$ data, but several branches in the $6 \times 6$ data. This difference is even more striking when comparing the $\sqrt{3} \times \sqrt{3}$ and the $6 \times 6$ dispersions shown in Figs. 2(c) and 2(e), especially between $\left(0.4-1.4 \AA^{-1}\right)$. The formation of a $6 \times 6$ superstructure on the $\sqrt{3} \times \sqrt{3}$ surface provides reciprocal lattice vectors that enable umklapp scattering of the $S_{1}$ band. The parts of the band structure that are due to umklapp scattering are denoted $\mathrm{Us}_{1}$. The $\bar{\Gamma}_{1}$-point of the $\sqrt{3} \times \sqrt{3} \mathrm{SBZ}$, at which the parabolic $S_{1}$ band is centered, coincides with a $\bar{\Gamma}_{2}$-point of an outer $6 \times 6$ SBZ. Due to umklapp scattering by $6 \times 6$ reciprocal lattice vectors, "replicas" of the parabolic $S_{1}$ band will appear in every $6 \times 6 \mathrm{SBZ}$. As a consequence of this, the single $S_{1}$ band of the $\sqrt{3} \times \sqrt{3}$ surface is accompanied by many $\mathrm{S}_{1}$ derived branches, $\mathrm{Us}_{1}$, originating from neighboring $6 \times 6 \mathrm{SBZs}$.

The Us 1 branches in Figs. 2(d) and 2(e) show various dispersions in different $k_{\square}$ ranges. In Fig. 2(d), one Us 1 branch is found at every $\bar{\Gamma}$-point of the $6 \times 6$ SBZs just below the Fermi level and disperses downward along $\bar{\Gamma}-\bar{K}-\bar{M}$. Another branch is found close to the $\bar{\Gamma}$-points and disperses downward along $\bar{\Gamma}-\bar{K}$ to $\approx-0.5 \mathrm{eV}$, see Fig. 2(d). Along the $\bar{\Gamma}-\bar{M}-\bar{\Gamma}$ direction of the 6×6 SBZs, see Fig. 2(e), a Us ${ }_{1}$ branch appears around the $\bar{M}$-point with an energy minimum at $0.35 \mathrm{eV}$ below $\mathrm{E}_{\mathrm{F}}$. From the $\bar{M}$-point it disperses upward toward $\bar{\Gamma}$, see Fig. 2(e).

There are other bands labeled $\mathrm{S}_{2}-\mathrm{S}_{6}$. In the $\bar{\Gamma}-\bar{K}-\bar{M}$ direction of the $6 \times 6 \mathrm{SBZ}$, the $\mathrm{S}_{2}$ band has a maximum energy at the $\bar{\Gamma}_{1}$ - point at $\approx 0.75 \mathrm{eV}$ below $E_{F}$, see Fig. $2(\mathrm{~d})$ and it disperses downward away from the $\bar{\Gamma}_{1}$-point. A similar band is also present on the $\sqrt{3} \times \sqrt{3}$ surface see Fig. 2(b). In Figs. 2(d) and 2(e), the $\mathrm{S}_{2}$ band shows a tiny dispersion toward $\bar{K}$ and $\bar{M}$-points with an energy minimum of $\approx-0.85 \mathrm{eV}$, at $\bar{\Gamma}_{1}$ and $\bar{\Gamma}_{2}$, respectively. However, at the outer $\bar{\Gamma}$-points, the band does not show a clear dispersion in either of these two directions. In the $\bar{\Gamma}-\bar{M}-\bar{\Gamma}$ direction of the $6 \times 6 \mathrm{SBZ}$, see Fig. 2(e), there are two more bands $S_{3}$ and $S_{4}$ at energies of $\approx 0.85$ and $\approx 1.4$ eV below $E_{F}$, respectively, in the $k_{\neg}$ range $0.6-1.1 \AA^{-1}$. These bands are similar to the $\mathrm{S}_{3}$ and $\mathrm{S}_{4}$ bands of the underlying $\sqrt{3} \times \sqrt{3}$ surface in the $\bar{\Gamma}-\bar{K}-\bar{M}$ direction of the SBZ as shown in Fig. 2(c). These bands are degenerate at $0.6 \AA^{-1}$ (i.e., the $\bar{K}$-point of $\sqrt{3} \times \sqrt{3}$ ) while the largest 
separation, $0.55 \mathrm{eV}$, occurs at $0.9 \AA^{-1}$ (i.e., the $\bar{M}$-point of $\sqrt{3} \times \sqrt{3}$ ). The $\mathrm{S}_{3}$ and $\mathrm{S}_{4}$ bands have been discussed in detail in Ref. 36.

(a)

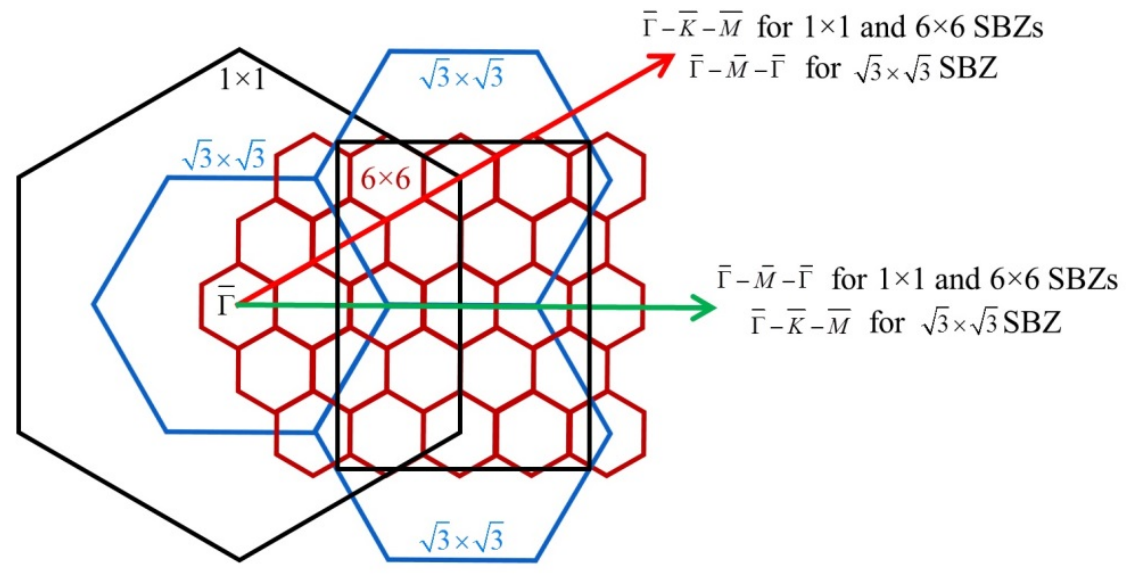

(b)

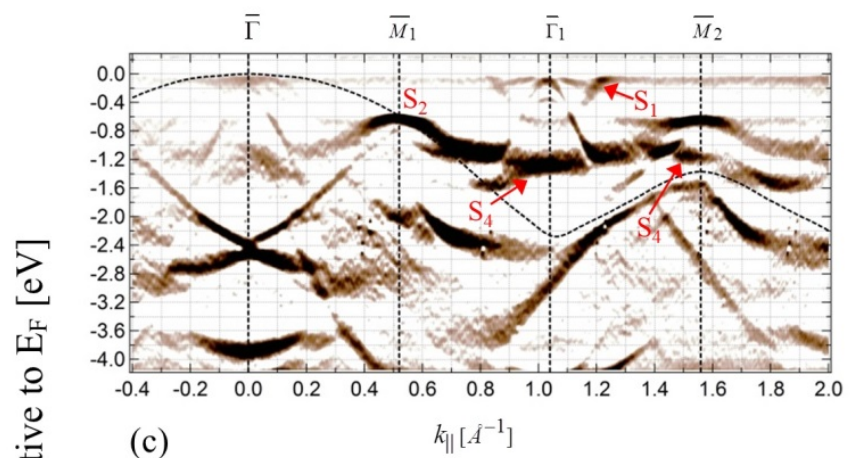

(c)



(d)

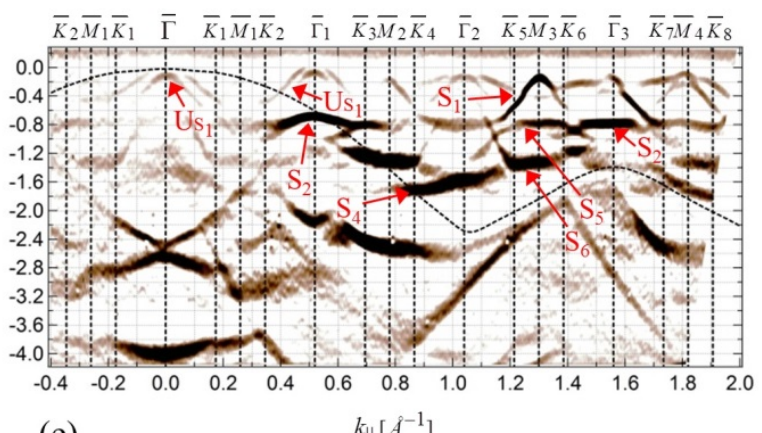

(e)

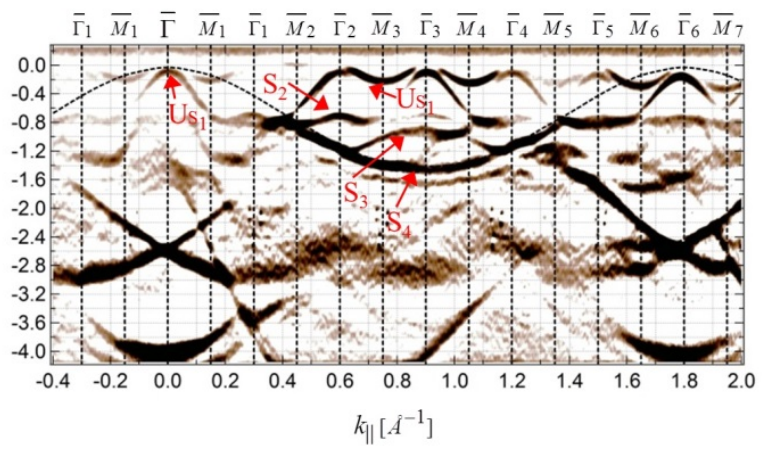

Fig. 2 (a) $1 \times 1, \sqrt{3} \times \sqrt{3}$ and $6 \times 6$ SBZs. The black rectangle shows the region mapped to obtain the 2D contour plots shown in Fig. 3. (b) and (c) ARPES results from the $\sqrt{3} \times \sqrt{3}$ surface along the high symmetry directions $\bar{\Gamma}-\bar{M}-\bar{\Gamma}$ (red line) and $\bar{\Gamma}-\bar{K}-\bar{M}$ (green line) of the $\sqrt{3} \times \sqrt{3} \mathrm{SBZ}$, respectively, measured with a photon energy of $30 \mathrm{eV}$ at RT. Vertical dashed lines in (b) and (c) mark high symmetry points of the $\sqrt{3} \times \sqrt{3}$ SBZ. (d) and (e) ARPES results from the $6 \times 6$ surface as in (b) and (c) but for the $\bar{\Gamma}-\bar{K}-\bar{M}$ (red line) and $\bar{\Gamma}-\bar{M}-\bar{\Gamma}$ (green line) directions of the $6 \times 6 \mathrm{SBZ}$, respectively. Vertical dashed lines in (d) and (e) mark high symmetry points of the $6 \times 6 \mathrm{SBZ}$. 
There is an obvious difference in energy and dispersion of the $S_{4}$ band on the $6 \times 6$ compared to the $\sqrt{3} \times \sqrt{3}$ surface, see Figs. 2(c) and 2(e). $S_{4}$ has a minimum energy of $-1.4 \mathrm{eV}$ at $0.9 \AA^{-1}$ instead of a local maximum at $-1.2 \mathrm{eV}$ in the case of $\sqrt{3} \times \sqrt{3}$. In addition, the band $\mathrm{S}_{3}$ is shifted downward in energy by $\approx 0.2 \mathrm{eV}$, cf. Figs. 2(c) and 2(e). In the $\bar{\Gamma}-\bar{K}-\bar{M}$ direction of the $6 \times 6 \mathrm{SBZ}$, the $\mathrm{S}_{4}$ band is located at an energy of $-1.6 \mathrm{eV}$ at the $\bar{\Gamma}_{2}$-point, see Fig. 2(d). This is similar to band $S_{4}$ in Fig. 2(b) at the $\bar{\Gamma}_{1}$ - point of the $\sqrt{3} \times \sqrt{3}$ surface, but the band is shifted down in energy by $\approx 0.3 \mathrm{eV}$. In addition, it is difficult to follow the dispersion of $\mathrm{S}_{4}$ in another $k_{\square}$ range. Along $\bar{\Gamma}-\bar{K}-\bar{M}$ of the $6 \times 6 \mathrm{SBZ}$, there are two more bands, $\mathrm{S}_{5}$ and $\mathrm{S}_{6}$ at -0.8 and $-1.4 \mathrm{eV}$, respectively, see Fig. 2(d). These bands do not show in any other $k_{\square}$ range.

Figure 3 presents constant energy contours obtained from the $\mathrm{Ag} / \mathrm{Ge}(111) 6 \times 6$ surface at RT with a photon energy of $30 \mathrm{eV}$. The bright features represent high photoemission intensity. The center of each panel of Fig. 3, i.e., $k_{x}=0.9 \AA^{-1}$ and $k_{y}=0 \AA^{-1}$, corresponds to the center of the fourth $6 \times 6 \mathrm{SBZ}\left(\bar{\Gamma}_{4}-\right.$ point) along $\bar{\Gamma}-\bar{M}-\bar{\Gamma}$ direction, see Fig. 2(a). In Figs. 3(a)-3(c) the contours at $0.2,0.3$, and $0.4 \mathrm{eV}$ below $\mathrm{E}_{\mathrm{F}}$ are shown, since at the lower energies the contours are very weak. Figure 3(a) shows complicated features inside each $6 \times 6$ SBZ resulting from the overlap of circles centered at the $\bar{\Gamma}$-points of neighboring $6 \times 6$ SBZs which appear more clearly in Figs. 3(b) and 3(c). The weak circles that start to show up at $-0.2 \mathrm{eV}$ in Fig. 3(a), increase in intensity going to the -0.3 to $-0.4 \mathrm{eV}$ constant energy contours. Each weak circle is centered at a $\bar{\Gamma}$-point of a $6 \times 6$ SBZ. The brighter circles at the top and bottom of the figures show cuts through the parabolic $S_{1}$ band of the $\sqrt{3} \times \sqrt{3}$ surface at the energies given above each figure. The centers of these two bright circles are $\bar{\Gamma}$-points of the $\sqrt{3} \times \sqrt{3}$ surface which coincide with $\bar{K}$-points of the $1 \times 1 \mathrm{SBZ}$. The weaker complicated pattern is made up of umklapp scattered circular $\mathrm{S}_{1}$ contours centered at various $6 \times 6 \bar{\Gamma}$-points. A similar situation has been reported for the $\mathrm{Ag} / \mathrm{Si}(111) \sqrt{21} \times \sqrt{21}$ surface when extra Au atoms had been deposited onto $\mathrm{Ag} / \mathrm{Si}(111) \sqrt{3} \times \sqrt{3}$ [21].

In order to sort out the shapes of the curves inside each $6 \times 6 \mathrm{SBZ}$, in Fig. 3(d), we have drawn circles tracing the experimental constant energy contours. Each circle (in red) is concentric with one of the $6 \times 6$ SBZs shown by the white hexagons. The schematic drawing in Fig. 3(e) illustrates the result of umklapp scattering. 

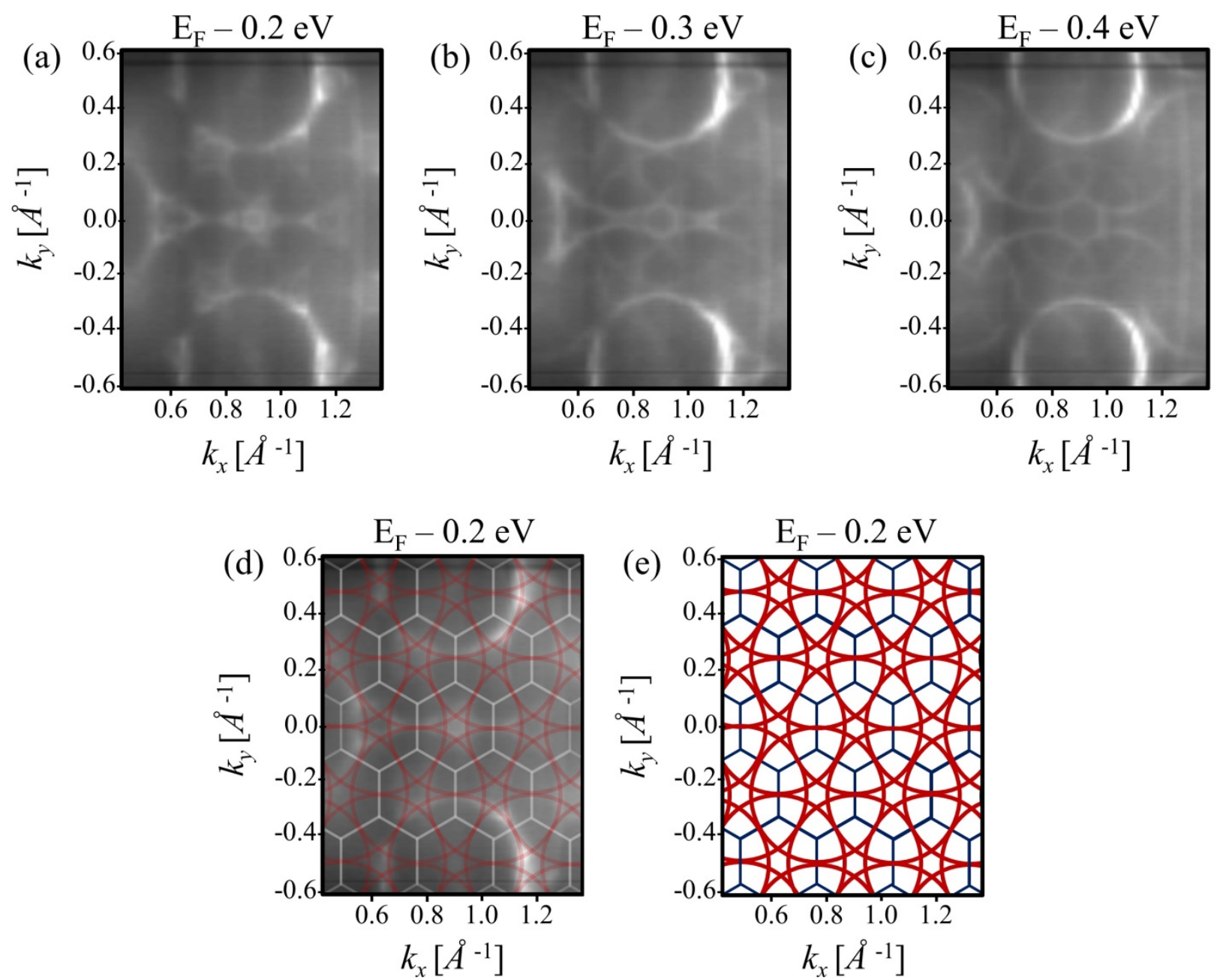

Fig. 3 (a)-(c) Gray scale images of two dimensional constant energy contours from the $\mathrm{Ag} / \mathrm{Ge}(111) 6 \times 6$ surface at RT. The contours, measured at a photon energy of $30 \mathrm{eV}$, are shown at three different energies as indicated. The center of each contour plot corresponds to the $\bar{\Gamma}_{4}$-point of the $6 \times 6$ SBZs (i.e., the $\bar{M}$ - point of the $1 \times 1 \mathrm{SBZ}$ ), see the black rectangle in Fig. 2(a). The contour plots are dominated by circles around the $\bar{\Gamma}$-point of each $6 \times 6$ SBZ. The weak circles are due to umklapp scattering, involving $6 \times 6$ reciprocal lattice vectors, of the bright circles originating from the $\sqrt{3} \times \sqrt{3}$ phase. (d) Circles (in red) are drawn that trace the experimental constant energy contours. (e) Schematic drawing summarizing the complex pattern of constant energy contours due to umklapp scattering of the $S_{1}$ band. Each circle is centered at a $\bar{\Gamma}$-point of a $6 \times 6 \mathrm{SBZ}$.

Figures 4(a) and 4(c) show schematic constant energy contours at an energy slightly above the Fermi level. The diameter of these circles is slightly larger than the experimental constant energy contours in Figs 3(a)-3(c), which were obtained at energies below the Fermi 
level. Red and green arrows indicate the $\bar{\Gamma}-\bar{K}-\bar{M}$ and $\bar{\Gamma}-\bar{M}-\bar{\Gamma}$ directions of the $6 \times 6 \mathrm{SBZ}$, respectively. In Figs. 4(b) and 4(d), the second derivative of the experimental umklapp branches (Us 1 ) are shown along the red and green arrows in Figs. 4(a) and 4(c), respectively. The dispersions of the umklapp branches can be explained by the contribution from the blue and green umklapp bands in 4(a) and those indicated in red and blue in Fig. 4(c). To guide the eye, the origins of the different umklapp branches in Fig. 4(b) and 4(d) are indicated by the color coded arrows.

a)

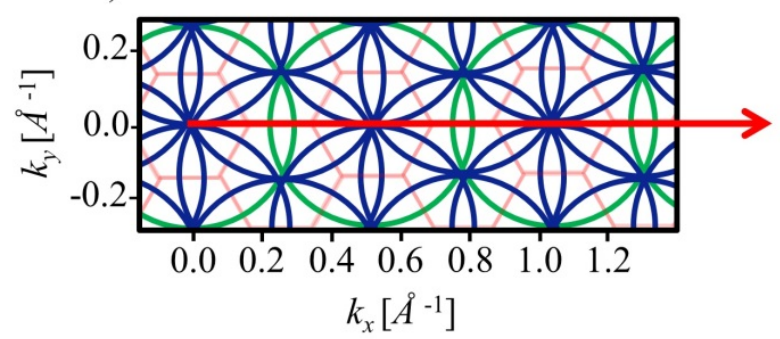

b)



c)

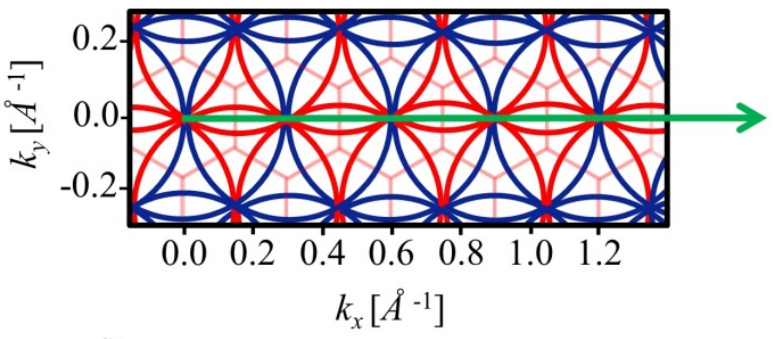

d) $\bar{\Gamma} \bar{M}_{1} \bar{\Gamma}_{1} \bar{M}_{2} \bar{\Gamma}_{2} \bar{M}_{3} \bar{\Gamma}_{3} \bar{M}_{4} \bar{\Gamma}_{4} \bar{M}_{5}$

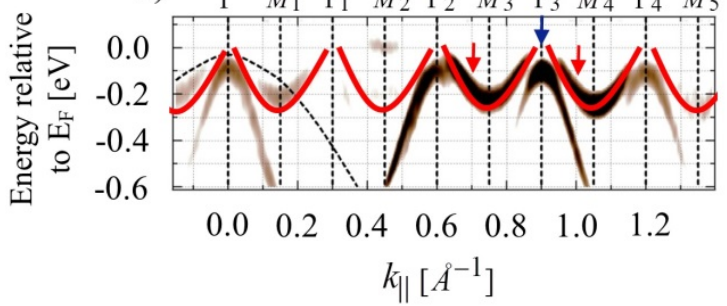

Fig. 4 (a) and (c) Schematic constant energy contours of the Ag/Ge(111)6×6 surface at an energy slightly above the Fermi level. The arrows in (a) and (c) indicate the $\bar{\Gamma}-\bar{K}-\bar{M}$ and $\bar{\Gamma}-\bar{M}-\bar{\Gamma}$ directions of $6 \times 6$ SBZ, respectively. (b) and (d) are the experimental band dispersions along the $\bar{\Gamma}-\bar{K}-\bar{M}$ and $\bar{\Gamma}-\bar{M}-\bar{\Gamma}$ directions from Figs. 2(d) and 2(e).

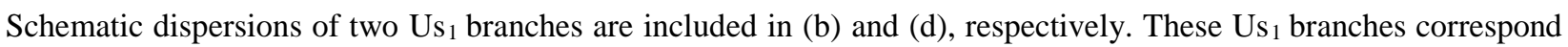
to the constant energy contours in (a) and (c) drawn in the same color.

The complicated surface band structure of the $6 \times 6$ surface can be understood in terms of umklapp scattering of the parabolic surface band $S_{1}$ originating from the underlying $\sqrt{3} \times \sqrt{3}$ structure. With the addition of $0.2 \mathrm{ML}$ of $\mathrm{Ag}$ to form the $6 \times 6$ superstructure the band minimum changed from 0.65 to $0.85 \mathrm{eV}$ below $\mathrm{E}_{\mathrm{F}}$. At the same time the extension in $k_{\square}$ increased. These changes can be seen by comparing the shape of $S_{1}$ in Fig. 2(b) with $S_{1}$ in Fig. 2(d). There is also a qualitative difference between $S_{1}$ of the $\sqrt{3} \times \sqrt{3}$ and $6 \times 6$ surfaces, i.e., $S_{1}$ crosses the Fermi level in the case of $\sqrt{3} \times \sqrt{3}$ but not for $6 \times 6$. In order to sort out the different umklapp branches of $S_{1}$, labeled $\mathrm{Us}_{1}$, 
we have drawn schematic representations of constant energy circles relevant for the $\bar{\Gamma}-\bar{K}-\bar{M}$ and $\bar{\Gamma}-\bar{M}-\bar{\Gamma}$ directions of $6 \times 6 \mathrm{SBZ}$ in Figs. 4(a) and 4(c). The schematic constant energy circles are drawn with a radius corresponding to an energy slightly above $\mathrm{E}_{\mathrm{F}}$, compared to the experimental curves in Fig. 3(a), for the sake of discussion.

The $\mathrm{S}_{1}$ band of the $6 \times 6$ surface, centered at the $\bar{\Gamma}_{2}$ - point along $\bar{\Gamma}-\bar{K}-\bar{M}$, is associated with the schematic constant energy circle centered at $k_{x}=1.05 \AA^{-1}$. A corresponding parabolic dispersion is drawn in Fig. 4(b). This schematic band, which mimics the $S_{1}$ band of the $\sqrt{3} \times \sqrt{3}$ surface, is drawn to a point slightly above the Fermi level. The umklapp replicas of the constant energy circles and schematic bands are also included in Figs. 4(a) and 4(b). The clear experimental dispersion around the $\bar{M}_{3}$-point deviates from what is expected based on the schematic bands. Instead of a band crossing, the experimental band structure has a maximum at the $\bar{M}_{3}$-point. This is indicative of a gap opening up at the $\bar{M}_{3}$-point. A similar gap was reported for the Au induced $\mathrm{Ag} / \mathrm{Si}(111) \sqrt{21} \times \sqrt{21}$ surface when umklapp branches of $\mathrm{S}_{1}$ from neighboring $\sqrt{21} \times \sqrt{21} \mathrm{SBZs}$ cross. In that case, the gap was found to be $110 \mathrm{meV}$ centered at an energy of approximately 0.28 $\mathrm{eV}$ below the Fermi level [21]. Since that gap was relatively small compared to the absolute energy position, the $S_{1}$ band from neighboring SBZs were also observed above the gap in the case of $\mathrm{Ag} / \mathrm{Si}(111) \sqrt{21} \times \sqrt{21}$. This is not the case for the $\mathrm{Ag} / \mathrm{Ge}(111) 6 \times 6$ surface.

A weak contribution from umklapp bands centered at $6 \times 6 \bar{\Gamma}$ - points above and below the $\mathrm{k}_{\mathrm{y}}=0$ line is also present. Since the two-dimensional dispersion of $S_{1}$ is not cut through the center in this case the dispersion will be shallower. A similar, but much clearer example of this, is shown in Figs. 4(c) and 4(d) along $\bar{\Gamma}-\bar{M}-\bar{\Gamma}$. The Us $\mathrm{s}_{1}$ branches with a minimum energy of $0.2 \mathrm{eV}$ at the $\bar{M}$ - points originate from bands centered at $\bar{\Gamma}$ - points above and below the $\mathrm{k}_{\mathrm{y}}=0$ line. Compared to the $\bar{\Gamma}-\bar{K}-\bar{M}$ direction, in Figs. 4(a) and 4(b), the $\mathrm{k}_{\mathrm{y}}=0$ line is further away from those $\bar{\Gamma}$ - points which results in a shallower dispersion. The overlap of the constant energy circles with their centers on the $\mathrm{k}_{\mathrm{y}}=0$ line is much larger along $\bar{\Gamma}-\bar{M}-\bar{\Gamma}$ than along $\bar{\Gamma}-\bar{K}-\bar{M}$ which gives rise to the other branches of Us ${ }_{1}$. As along $\bar{\Gamma}-\bar{M}-\bar{\Gamma}$, the Us ${ }_{1}$ dispersion shows maxima instead of band crossings when the Us ${ }_{1}$ branches from neighboring $6 \times 6$ SBZs meet, see Fig. 4(d). The presence of gaps in the surface band structure is confirmed in both high symmetry directions. 


\section{Conclusions}

It has been shown that the general appearance of the complex surface band structure of the $6 \times 6$ phase is a combination of the $\sqrt{3} \times \sqrt{3}$ surface band structure and umklapp replicas of that band structure. In similarity with observations on the $\sqrt{21} \times \sqrt{21}$ surface prepared by adding Au to the $\mathrm{Ag} / \mathrm{Si}(111) \sqrt{3} \times \sqrt{3}$ surface [21], gaps in the surface band structure open up in regions of the two-dimensional k-space where the umklapp branches of the $\mathrm{S}_{1}$ bands cross each other. The Fermi level is located within these gaps, which is in contrast to the Au induced $\sqrt{21} \times \sqrt{21}$ surface of $\mathrm{Ag} / \mathrm{Si}(111)$, where the upper edge of the gap is about $0.2 \mathrm{eV}$ below the $\mathrm{E}_{\mathrm{F}}$. The complex pattern of constant energy contours played a crucial role in the identification of the various dispersion branches observed in the ARPES data.

\section{Acknowledgements}

Technical support from Dr. Johan Adell and Dr. T. Balasubramanian at MAX-lab is gratefully acknowledged. Financial support of the research work was provided by the Swedish Research Council (VR).

\section{References}

[1] Norio Sato, Tadaaki Nagao, and Shuji Hasegawa, Phys. Rev. B 60 (1999) 16083.

[2] Eli Rotenberg, H. Koh, K. Rossnagel, H.W. Yeom, J. Schäfer, B. Krenzer, M.P. Rocha, and S.D. Kevan, Phys. Rev. Lett. 91(2003) 246404, and references therein.

[3] J.L. McChesney, J.N. Crain, V. Pérez-Dieste, Fan Zheng, M.C. Gallagher, M. Bissen, C. Gundelach, and F.J. Himpsel, Phys. Rev. B 70 (2004) 195430.

[4] Harumo Morikawa, Iwao Matsuda, and Shuji Hasegawa, Phys. Rev. B 77 (2008) 193310.

[5] Ryota Niikura, Kan Nakatsuji, and Fumio Komori, Phys. Rev. B 83 (2011) 035311.

[6] P. Höpfner, J. Schäfer, A. Fleszar, S. Meyer, C. Blumenstein, T. Schramm, M Heßmann, X. Cui, L. Patthey, W. Hanke, and R. Claessen, Phys. Rev. B 83 (2011) 235435.

[7] D. Grozea, E. Bengu, L.D. Marks, Surf. Sci. 461 (2000) 23.

[8] E.L. Bullock, G.S. Herman, M. Yamada, D.J. Friedman, and C.S. Fadley, Phys. Rev. B 41 (1990) 1703.

[9] H.M. Zhang, Kazuyuki Sakamoto, and R.I.G. Uhrberg, Phys. Rev. B 70 (2004) 245301.

[10] H.M. Zhang, T. Balasubramanian, and R.I.G. Uhrberg, Phys. Rev. B 63 (2001) 195402. 
[11] Iwao Matsuda, Toru Hirahara, Mitsuru Konishi, Canhua Liu, Harumo Morikawa, Marie D’angelo, Taichi Okuda, Toyohiko Kinoshita and Shuji Hasegawa, Phys. Rev. B 71 (2005) 235315.

[12] G. Le Lay, V. Yu. Aristov et al., Surf. Sci. 307-309 (1993) 280.

[13] H.H. Weitering, and J.M. Carpinelli, Surf. Sci. 384 (1997) 240.

[14] D.J. Spence, S. P. Tear, Surf. Sci. 398 (1998) 91.

[15] L.S.O. Johansson, E. Landemark, C.J. Karlsson, and R.I.G. Uhrberg, Phys. Rev. Lett. 63 (1989) 2092.

[16] Y.G. Ding, C.T. Chan, and K.M. Ho, Phys. Rev. Lett. 67 (1991) 1454.

[17] H. Huang, H. Over, J. Quinn, F. Jona, and S.Y. Tong, Phys. Rev. B 49 (1994) 13483.

[18] Yuji Nakajima, Sakura Takeda, Tadaaki Nagao, Xiao Tong, and Shuji Hasegawa, Phys.

Rev. B 56 (1997) 6782.

[19] Xiao Tong, Satoru Ohuchi, Norio Sato, Takehiro Tanikawa, Tadaaki Nagao, Iwao Matsuda, Yoshinobu Aoyagi, and Shuji Hasegawa, Phys. Rev. B 64 (2001) 205316.

[20] R.I.G. Uhrberg, H.M. Zhang, T. Balasubramanian, E. Landemark, and H.W. Yeom, Phys. Rev. B 65 (2002), 081305(R).

[21] J.N. Crain, K.N. Altmann, C. Bromberger, and F.J. Himpsel, Phys. Rev. B 66 (2002) 205302. [22] Iwao Matsuda, Harumo Morikawa, Canhua Liu, Satoru Ohuchi, Shuji Hasegawa, Taichi Okuda, Toyohiko Kinoshita, Carlo Ottaviani, Antonio Cricenti, Marie D’angelo, Patrick Soukiassian, and Guy Le Lay, Phys. Rev. B 68 (2003) 085407.

[23] H.M. Zhang, R.I.G. Uhrberg, Surf. Sci. 546 (2003) L789.

[24] J.N. Crain, M.C. Gallagher, J.L. McChesney, M. Bissen, and F.J. Himpsel, Phys. Rev. B 72 (2005) 045312.

[25] Canhua Liu, Iwao Matsuda, Rei Hobara, and Shuji Hasegawa, Phys. Rev. Lett. 96 (2006) 036803.

[26] H.M. Zhang, R.I.G. Uhrberg, Phys. Rev. B 74 (2006) 195329.

[27] L.-W. Chou, H.C. Wu, Y.-R. Lee, J.-C. Jiang, C. Su, and J.-C. Lin, J. Chem. Phys. 131 (2009) 224705.

[28] Xiao Tong, Shuji Hasegawa, and Shozo Ino, Phys. Rev. B 55 (1997) 1310.

[29] Xiao Tong, Chun Sheng Jiang and Shuji Hasegawa, Phys. Rev. B 57 (1998) 9015.

[30] H.M. Zhang, Kazuyuki Sakamoto, and R.I.G. Uhrberg, Phys. Rev. B 64 (2001) 245421. 
[31] Canhua Liu, Iwao Matsuda, Harumo Morikawa, Hiroyuki Okino, Taichi Okuda, Toyohiko Kinoshita, and Shuji Hasegawa, Jpn. J. Appl. Phys. 42 (2003) 4659.

[32] Canhua Liu, Iwao Matsuda, Shinya Yoshimoto, Taizo Kanagawa, and Shuji Hasegawa, Phys. Rev. B 78 (2008) 035326.

[33] H.M Zhang, T Balasubramanian, R.I.G Uhrberg, Appl. Surf. Sci. 175-176 (2001) 237.

[34] H.M. Zhang, Kazuyuki Sakamoto, and R.I.G. Uhrberg, Surf. Sci. 532-535 (2003) 934.

[35] H.M. Zhang, R.I.G. Uhrberg, Appl. Surf. Sci. 212-213 (2003) 353.

[36] Hafiz M. Sohail, R.I.G. Uhrberg, Surf. Sci. 625 (2014) 23-29. 